Steering of sub- GeV charged particle beams by use of reflections in thin crystal targets

This article has been downloaded from IOPscience. Please scroll down to see the full text article.

2012 JINST 7 P03008

(http://iopscience.iop.org/1748-0221/7/03/P03008)

View the table of contents for this issue, or go to the journal homepage for more

Download details:

IP Address: 137.138.93.221

The article was downloaded on $23 / 07 / 2013$ at $15: 22$

Please note that terms and conditions apply. 


\title{
Steering of sub- GeV charged particle beams by use of reflections in thin crystal targets
}

\author{
S. Bellucci, ${ }^{a, 1}$ Yu.A. Chesnokov ${ }^{b, c}$ P.N. Chirkov, ${ }^{b}$ G. Giannini, ${ }^{a}$ V.A. Maisheev ${ }^{b, c}$ \\ and I.A. Yazynin ${ }^{b, c}$ \\ ${ }^{a}$ INFN — Laboratori Nazionali di Frascati, \\ Via E. Fermi 40, 00044 Frascati, Roma, Italy \\ ${ }^{b}$ Institute of High Energy Physics, \\ Moscow Region, RU-142281 Protvino, Russia \\ ${ }^{c}$ CERN - European Organization for Nuclear Research, \\ CERN CH-1211 Geneve 23, Switzerland \\ E-mail: bellucci@lnf.infn.it
}

\begin{abstract}
The phenomenon of deflection of a charged particle beam due to channeling in a bent crystal has been well investigated and successfully applied for beam extraction at high-energy accelerators, for energies about $10 \mathrm{GeV}$ and higher. However, it is of a big practical interest to consider the task of bending and extracting charged particles with energies below $1 \mathrm{GeV}$, for example, for production of ultrastable beams of low emittance for medical and biological applications. However, for low energy, i.e. below $1 \mathrm{GeV}$, the bent crystal channeling is not efficient. That motivates us to consider in this article an other crystal technique, based on thin straight crystal targets, as elements for the extraction and collimation of the circulating beam in an accelerator ring. The main advantages of reflection in straight crystals, in comparison with bent crystal channeling, consist in the small length of straight crystals along the beam, that reduces the amount of nuclear interactions and improves the background.
\end{abstract}

KEYWORDS: Instrumentation for particle-beam therapy; Instrumentation for particle accelerators and storage rings - low energy (linear accelerators, cyclotrons, electrostatic accelerators); Beam dynamics; Beam-line instrumentation (beam position and profile monitors; beam-intensity monitors; bunch length monitors)

\footnotetext{
${ }^{1}$ Corresponding author.
} 


\section{Contents}

1 Introduction 1

2 Principle of the method of particle beam steering by reflections in short crystals 1

3 Experimental feasibility of beam deflection by thin crystal targets 2

4 Conclusion $\quad 5$

\section{Introduction}

The phenomenon of deflection of a charged particle beam due to channeling in a bent crystal is well investigated and successfully applied for the extraction of beam in high-energy accelerators, at energies of about $10 \mathrm{GeV}$ and higher (see for example ref. [1-3]).

However, the task of bending and extraction of charged particles with energies below $1 \mathrm{GeV}$ is particularly interesting, e.g. for production of ultrastable beams of low emittance for medical and biological applications [4]. The innovative technique based on crystals could significantly reduce the costs for setting up a beam line dedicated to medical beam creation.

There exists a big experimental problem in steering of such energy beams which is connected with small sizes of bent crystal samples. The think is that characteristic parameter $L_{d}$, called dechanneling length is relatively small for low energy. For $E=500 \mathrm{MeV}$, we have $L_{d}=0.4 \mathrm{~mm}$ [1]. With usual channeling bent crystals (about $1 \mathrm{~mm}$ in length) only $10 \%$ efficiency was achieved for the deflection of sub-GeV energy particles [5] in beam line.

More serious problems arise when a circulating beam is extracted from a circular accelerator, since crystals required for this task must have large transverse dimensions exceeding the crystal length. In addition, the bending angle of the crystal must be $\sim 1$ mrad in order that the deflected beam could be easily separated from the circulating one in the energy region below $1 \mathrm{GeV}$. Potentially, quasimosaic bent crystals [6] or thin flat crystals [7, 8] can be used for this purpose; nevertheless, in either case, the angle of deflection of particles must be increased several times.

\section{Principle of the method of particle beam steering by reflections in short crystals}

The method is based on particle reflection from very thin straight crystal plates [7], the thickness of which is equal to an odd number of half-waves of particle path oscillations during channeling: $L=(2 n+1) / 2 \times \lambda$, where $\lambda=\pi d / \theta c, d=2.3 \AA$ - interplanar distance in silicon. It should be noted here that an analogous effect in axial crystal channeling has been reported [9]. It is characterized by the pronounced maxima of zero-degree yield corresponding to an odd number of half-waves of oscillations of axially channelled particles. The effect of particle deflection by a thin crystal is shown in figure 1 . 


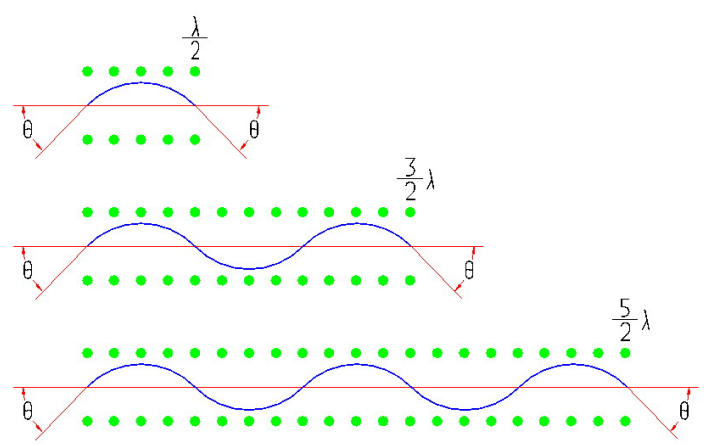

Figure 1. Effect of particle trajectory bending in a thin crystal.

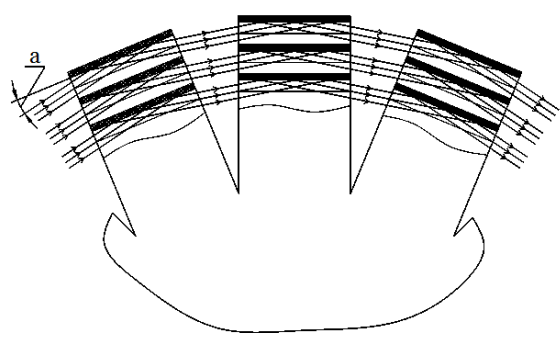

Figure 2. Fan-type reflector for bending of particle beam with use of thin straight crystals. Reflection of trajectories of particles from nuclear planes is schematically shown.

This means that, e.g., the optimum crystal length must be about $1 \mu \mathrm{m}$ for $500 \mathrm{MeV}$ particles. The maximal reflection angle in a single optimum length silicon plate is approximately equal to the doubled critical angle for channeling $\theta_{c}=\left(2 U_{o} / p v\right)^{1 / 2}$, where $U_{o} \sim 20 \mathrm{eV}$ is the planar channel value in silicon; $p$ and $v$ are the momentum and velocity of the incident particle, respectively. But for real accelerator applications this angle of deflection is not sufficient. Several oriented crystal plates unfolded like a fan (see ref. [10]) can be used to increase the deflection angle (figure 2).

To ensure optimum beam deflection in this construction, each successive crystal is deflected through angle about $\theta_{c}$. As a result, the total deflection angle may be as large as $\theta c \times N$, where $N$ is the number of crystal plates.

\section{Experimental feasibility of beam deflection by thin crystal targets}

The first experimental testing of the fan-like reflector was made in [10] in $50 \mathrm{GeV}$ circulating beam. Good efficiency of deflection, up to $80 \%$ was achieved, but deflection angle was limited because of non-optimal construction of first crystal device. For optimization of crystal targets we propose a research about the deflection of particles in the Beam Test Facility of the LNF INFN, where a particle beam with energies of $50-700 \mathrm{MeV}$ is readily available [5]. The necessary experimental facility contains a remote controlled goniometer for installation of crystal targets, beam collimator and detector. The scheme of experimental setup is shown in figure 3 


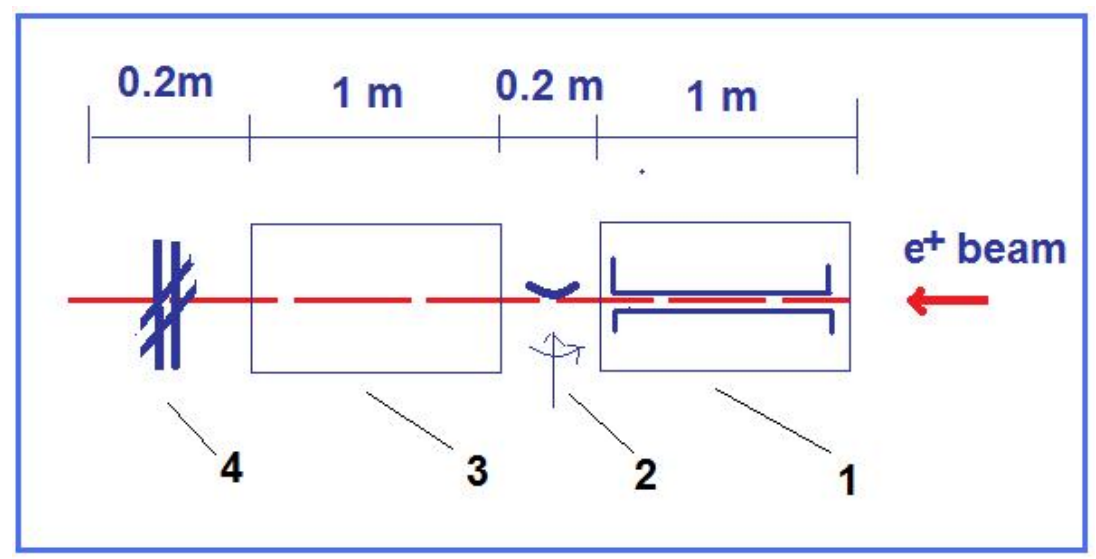

Figure 3. 1: steel collimator in vacuum pipe; 2: crystal sample in goniometer; 3: drift space in vacuum about 1 meter long; 4: microstrip detector with 50 micron resolution.

It is well-known that only particle beams with small angular divergence within the limits of a critical channeling angle can steer with the help of straight crystals ([1], page 34). Low emittance e+ beam is obtained using special iron collimator. The horizontal emittance of the beam $\varepsilon \approx 0.5 \mathrm{~mm} \times 0.5 \mathrm{mrad}$ and $\varphi \approx \pm 0.25 \mathrm{mrad}$ are achieved. So, in our case the ratio $\left(\theta_{c} / \varphi\right)<1$ is achieved ( $\theta_{c}$ is about 360 microrad for $300 \mathrm{MeV}$ particles), which is appropriate for efficient particle deflection observation. Efficiency of beam deflection in our case is understood as a fraction of the particles bend by the crystal device on angle more than $1 \mathrm{mrad}$ in relation to intensity of all the beam falling on a crystal.

First of all, the opportunity of beam bending by a fan-like deflector will be investigated. Preliminary Monte Carlo calculations of $300 \mathrm{MeV}$ positron beam deflection with the help of the fanlike reflector were performed by the code SCRAPER [11]. In simulations a narrow beam with zero angular divergence with an incident angle $\vartheta=-\alpha / 2 n$ relative to first fan crystal is assumed. The turn of the next plates of a fan is about $\vartheta=-\alpha / n$. The angle $\alpha=1.4 \mathrm{mrad}$ here is the total beam bending, and $\mathrm{n}$ is the number of crystal plates. A good efficiency was found for a reflector consisting of five silicon single crystals of the (110) planar orientation (see figure 4). In this case $52 \%$ of beam is deflected on an angle larger than $1 \mathrm{mrad}$.

In addition to the bending of particle beams, this crystal technique can be used for shaping the beam profiles for different applications. In this case particle interactions with the array of short straight crystals give a new possibility of beam angular divergence regulation.

The task of beam profile shaping is needed for example in radiation therapy. Usually transversal filters (the special volume collimators) are used for obtaining a beam with given shape and size, for producing a fixed dose in cancer tumor therapy applications. The shortcoming of this method is the necessity to utilize rather thick targets, due to the resulting additional harmful background and intensity decreasing of useful beam taking place. Besides, the beam shaping in one plane brings distortion in the other plane (due to multiple scattering, mainly). The new method of beam shaping based on a sequence of thin crystals, allows us to change the angular distribution in one plane without influencing the angular distribution in another plane. Due to the small target thickness (of about several microns), additional secondary backgrounds as a result of beam interaction with the 


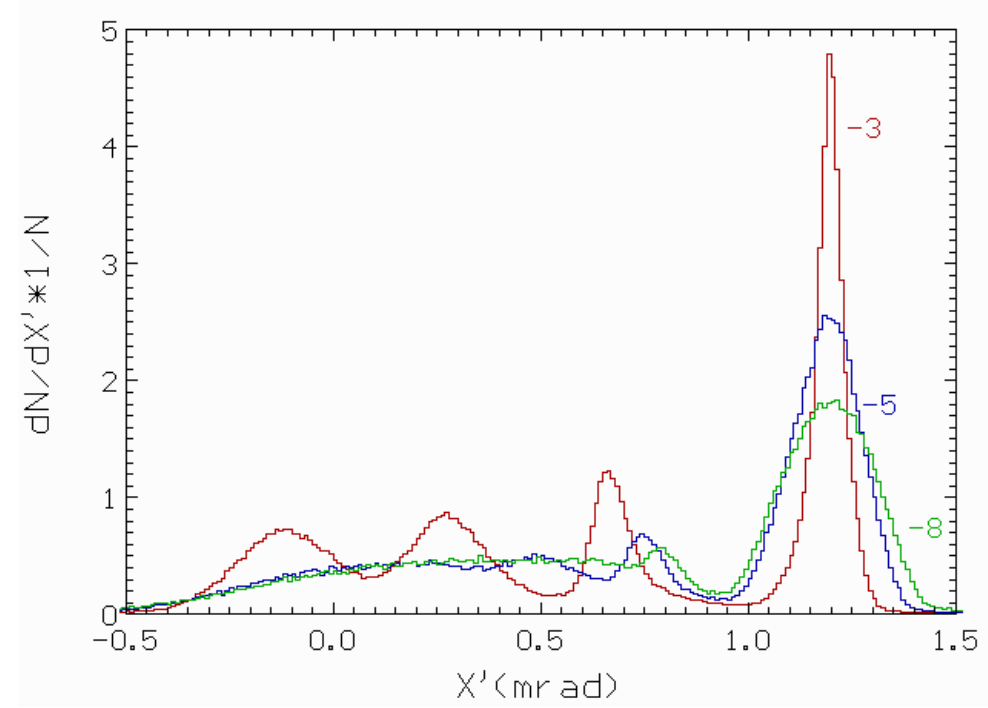

Figure 4. Deflection of the beam with the help of fan reflector consisting from $n=3,5,8$ single crystals.

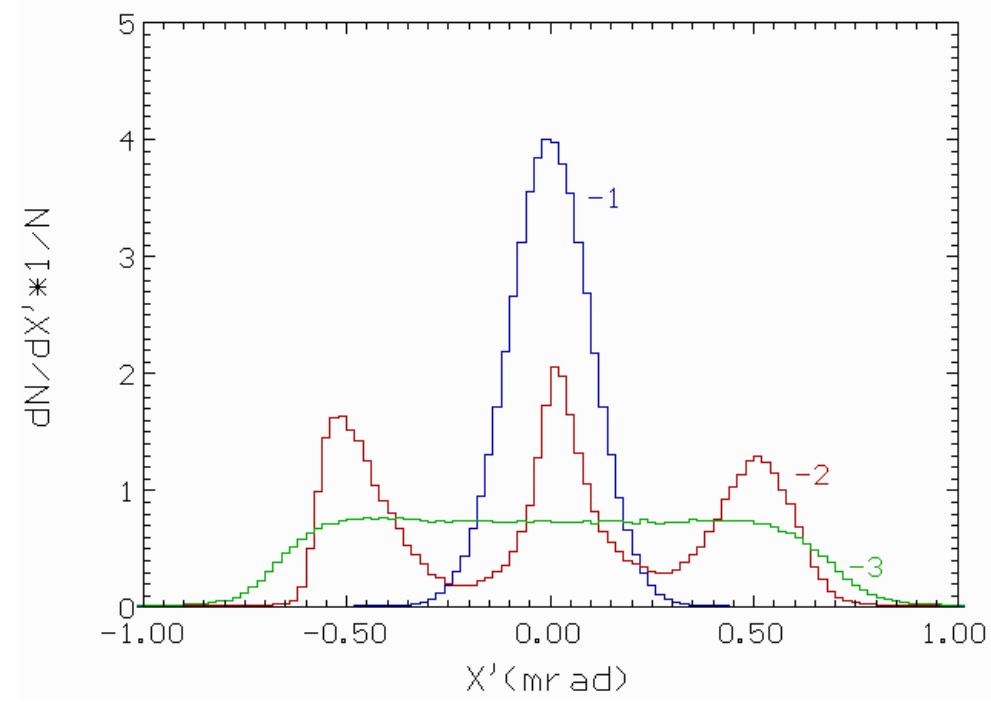

Figure 5. The samples of initial angular divergence (curve 1) shaping with the help of two (curve 2) and four (curve 3) crystals. The turn of the crystal plates in array is about $\pm 0.3 \mathrm{mrad}$ in this case.

target there are absent. We carried out computer simulations for the investigation of possibilities of creating such crystal targets. Figure 5 illustrates the shaping of the flat angular distribution of particle beam at $300 \mathrm{MeV}$ energy from the initially Gaussian distribution.

One can see from the figure that, in the case of usage of the four silicon single crystals of the (110) orientation, the resulting angular distribution is 5 times wider initial distribution and has a plateau. At a definite distance downstream the crystal fan the particle angles transform to coordinates and we may get the beam with uniform angular distribution and required size. 


\section{Conclusion}

In the paper a method of beam steering by fan-like crystal reflector and its theoretical and experimental feasibility for sub-GeV energy are discussed. This method yields a good perspective for the creation of cheap medical beams with energy below $1 \mathrm{GeV}$. Advantages of reflection in straight crystals, in comparison with bent crystal channeling, consist in the small length of straight crystals along the beam that reduces the amount of nuclear interactions and improves the background.

Very interesting research developments will probably occur with the application of nanoporous materials and aligned nanostructures. The realization of such special targets is in a stage of development [12-14].

\section{Acknowledgments}

This work was supported in part by Transnational Access to Research Infrastructure (TARI) INFN — Laboratori Nazionali di Frascati, HadronPhysics2 - Integrating Activity, Contract No. 227431, 01/01/2009 - 31/12/2011. We thank Srdjan Petrović for pointing out the analogy of certain aspects of figure 1 with ref. [8].

\section{References}

[1] V.M. Biryukov, Yu.A. Chesnokov and V.I. Kotov, Crystal channeling and its application at high-energy accelerators, Springer, U.S.A. (1997).

[2] W. Scandale et al., High-efficiency volume reflection of an ultrarelativistic proton beam with a bent silicon crystal, Phys. Rev. Lett. 98 (2007) 154801.

[3] N.V. Mokhov A., et al., Crystal collimation studies at the tevatron (T-980), Int. J. Mod. Phys. A 25 (2012) S98 [FERMILAB-CONF-09-173-APC];

V.M. Biryukov and S. Bellucci, Crystal bending of the LHC beam for in situ calibration of ATLAS and CMS calorimeters, Nucl. Instrum. Meth. B 252 (2006) 7;

H.H. Andersen, S. Bellucci and V.M. Biryukov, Edtorial, Nucl. Instrum. Meth. B 252 (2006) 1;

S. Bellucci and V.M. Biryukov, The future looks bright for particle channelling, CERN Cour. 46 (2006) 37;

H.H. Andersen, S. Bellucci and V.M. Biryukov, Editorial, Nucl. Instrum. Meth. B 234 (2005) 1.

[4] V.M. Biryukov, S. Bellucci and V. Guidi, Channeling technique to make nanoscale ion beams, Nucl. Instrum. Meth. B 231 (2005) 70;

S. Bellucci, New radiation sources from channeling in micro- and nano-structures, Proc. SPIE $\mathbf{5 8 5 2}$ (2005) 276;

S. Bellucci and S.B. Dabagov, On x-ray channelling in microcapillaries and nanocapillaries, J. Phys. Condensed Matter 15 (2003) 3171.

[5] S. Bellucci et al., Using a deformed crystal for bending a sub-GeV positron beam, Nucl. Instrum. Meth. B 252 (2006) 3.

[6] Yu.M. Ivanov, A.A. Petrunin and V.V. Skorobogatov, Observation of the elastic quasi-mosaicity effect in bent silicon single crystals, JETP Lett. 81 (2005) 99.

[7] A. Taratin et al., Ultrathin crystal scatterer for the SSC beam extration system, Preprint SSCL-545 (1991). 
[8] S. Strokov et al., Electron beam deflection with channeling in a silicon crystal at the REFER electron ring, Nucl. Instrum. Meth. B 252 (2006) 16.

[9] L. Mileti, S. Petrović and N. Nešković, Decay of zero-degree focusing of channeled ions, Nucl. Instrum. Meth. B 115 (1996) 337; Energy dependence of zero-degree focusing of channeled ions, Radiat. Eff. Defect. S. 141 (1997) 235.

[10] A.G. Afonin et al., Crystal-assisted beam extraction and collimation at the U-70 circular accelerator, Instrum. Exp. Tech. 54 (2011) 1 [arXiv:1102.2711].

[11] I.I. Degtyarev, O.A. Liashenko and I.A. Yazynin, Applications of the coupled SCRAPER-RTS\&T code in radiation therapy, in the proceedings of the EPAC 2000, June 26-30, Vienna, Austria (2000).

[12] S. Bellucci, Nanotubes for particle channeling, radiation and electron sources, Nucl. Instrum. Meth. 234 (2005) 57;

V.M. Biryukov and S. Bellucci, Nanostructures versus crystals in particle channeling, Nucl. Instrum. Meth. B 234 (2005) 99;

S. Bellucci et al., Channeling of high energy beams in nanotubes, Nucl. Instrum. Meth. B 202 (2003) 236;

S. Bellucci et al., Making micro- and nano-beams by channeling in micro- and nano-structures, Phys. Rev. ST Accel. Beams 6 (2003) 033502 [physics/0209057];

S. Bellucci, V.M. Biryukov and A. Cordelli, Channeling of high-energy particles in a multi-wall nanotube, Phys. Lett. B 608 (2005) 53 [physics/0501006];

V.M. Biryukov and S. Bellucci, Studies of nanotube channeling for efficient beam scraping at accelerators, Nucl. Instrum. Meth. B 230 (2005) 619.

[13] E. Dervishi et al., High-aspect ratio and horizontally oriented carbon nanotubes synthesized by RF-cCVD, Diamond Relat. Mater. 19 (2012) 67.

[14] X.Y. Zhang et al., Template synthesis of high-density carbon nanotube arrays, J. Cryst. Growth 223 (2001) 306. 